\title{
Robust Solar Sail Trajectory Control for Large Pre-Launch Modeling Errors
}

\author{
L. Rios-Reyes and D. J. Scheeres
}

\begin{abstract}
Solar sail missions will be designed for specific propulsion models of a sail, however due to uncertainties in sail performance and degradation these models must always be conservative and provide excess thrust. It is not simple to shed excess thrust on a solar sail, and it may not be feasible or wise to continually re-optimize and re-target the trajectory based on the current performance of the sail. We present an alternate control methodology that dumps excess thrust by forcing the sail to "orbit" about the nominal solution in a quasi-circular motion constrained to lie in the plane perpendicular to the nominal thrust direction. The excess thrust is balanced by the centripetal acceleration necessary to maintain the motion. A feedback control scheme can be used to asymptotically stabilize this orbital motion and an estimation strategy to track changing sail performance parameters. Implementation strategies for this approach are also discussed.
\end{abstract}

\section{Introduction}

Solar sail missions must be designed for a specific sail propulsion model. However, due to the complexity of solar sails and difficulties in providing precise predictions of their performance, our ability to precisely predict the sail acceleration performance is inherently limited. To account for this, trajectory design of a sail mission must be performed with a conservative propulsion model that provides adequate margin for uncertainties in the sail performance. Specifically, it is important that the sail provide a guaranteed minimum amount of thrust to ensure that the chosen mission can be flown. Due to this, however, the actual sail will most likely always have more thrust than the original mission planned for. Due to the nature of solar sails, this excess thrust cannot be easily modulated and will fundamentally change the trajectory of the sail, making it extremely difficult to achieve mission targets. While it is possible to estimate the actual sail propulsion after deployment and redesign the sail trajectory, this constitutes an open and continual design process that may not always be feasible to implement or converge on the proper target. Similarly, as the sail surface suffers from degradation ${ }^{1}$ throughout the mission, the thrust will change continuously and, if the maximum thrust of the sail is being used, constant re-optimization and redesign of the trajectory would be necessary.

In this paper we consider a more consistent methodology for dealing with an excess of sail performance that does not require a continually redesigned trajectory as the sail properties change over time. Our approach will allow a sail to follow a nominal mission design so long as the sail provides an excess of thrust, which will be the nominal situation for any sail mission. Ideally, our approach can be applied to any trajectory, and will allow the sail to follow a nominal mission profile. In this paper we only consider the simpler problem of maintaining a sail at a specific sub- $L_{1}$ equilibrium point location. Future work will generalize this to tracking an arbitrary trajectory.

Under the assumption that the sail is producing more thrust than is necessary to maintain its nominal position (or to follow its nominal trajectory), the problem essentially becomes how to shed the excess thrust of the sail. The opposite situation, if the sail does not produce as much thrust as is needed to follow the nominal trajectory, can be catastrophic and lead to mission failure. Due to this, we assume that the trajectory will always be designed with a conservative model of the sail, and hence that there will always

\footnotetext{
${ }^{*} \mathrm{Ph}$. D. Candidate, Department of Aerospace Engineering, The University of Michigan, 1320 Beal Avenue, Ann Arbor, MI 48105-2140, leonelr@umich.edu, Student Member AIAA.

${ }^{\dagger}$ Associate Professor, Department of Aerospace Engineering, The University of Michigan, 1320 Beal Avenue, Ann Arbor, MI 48105-2140, scheeres@umich.edu, Member AAS, Associate Fellow AIAA.
} 
be a nominal amount of excess thrust to be dumped. It is important to note that this is not "inefficient" so long as the sail reaches its desired goal, as there is no fuel penalty for dumping excess thrust - at most there may be a time penalty. Our approach does not rule out the possibility of re-optimizing the sail trajectory to achieve its goals in a shorter time, however it allows the re-optimization process to be performed independent of the real-time performance of the sail.

Consider our example of maintaining a specific sub- $L_{1}$ equilibrium point location. In general, if the sail has less thrust than the design performance, it will not be possible for it to maintain this position and the mission cannot be achieved. If it has precisely the design performance, then it is possible to maintain the equilibrium point and even perform control maneuvers to maintain itself close to this location, accounting for errors in initial condition and other factors. ${ }^{2}$ If the sail has more thrust than its design performance, however, it becomes difficult to maintain the target location without actively modulating the total surface area of the sail. Such modulation would be a difficult and complex procedure to carry out with the sail structure.

The main problem in this situation is that the sail has excess thrust. A simple solution is to orient the sail so that it provides the appropriate thrust level along the line connecting the two primaries (the line of syzygies), and "dump" the excess thrust along the direction perpendicular to this line. In a short time, of course, the sail would veer off course and leave the equilibrium. A simple fix would be to switch, or dither, the sail back and forth - maintaining the proper thrust along the line of syzygy and producing a net zero thrust perpendicular to this line. Such an approach may work conceptually, but could not be easily implemented. Following this line of thought, however, we find a simple approach that can essentially achieve this goal of maintaining the appropriate thrust vector and forcing the excess thrust to average out to zero.

To accomplish this task we force the sail to "orbit" the equilibrium point (or a nominally moving point along a trajectory) in a small semicircular orbit centered on this nominal solution. Instead of forcing the sail to dither back and forth, however, we have the sail follow a semicircular path about the nominal solution that has a centripetal acceleration equal to the amount of excess thrust generated after the sail is oriented to provide the nominal thrust. Thus, the sail will follow the nominal trajectory while performing a controlled "orbit" about the trajectory. For a given value of excess thrust there is some freedom in designing this orbit, as the centripetal acceleration depends on both the radius of this orbit and the angular rate.

This control approach can be implemented by inducing the sail to rotate at the same rate as it "orbits" the nominal solution. Thus the attitude of the sail is fixed such that the excess thrust is always pointed towards the nominal solution. There may be some control issues with this approach if the sail is being forced to rotate about a non-principal axis. It should be possible, however, for the attitude control system to ensure that the sail maintains this attitude.

We have been able to implement this control for our example problem of maintaining a specified sub$L_{1}$ equilibrium point. Additionally, by modulating the angle that the sail makes with the sun-line with a closed-loop feedback control we are able to stabilize the sail so that it maintains a constant location along the line of syzygies and follows a small circular orbit about the equilibrium location. Using a PD-controller and the use of an adaptive control technique allows the sail to update its parameters to ensure that the same location is maintained as the sail properties change with age. In this paper we develop them and test them in a variety of situations to ensure their robustness.

\section{Circular Restricted Three Body Problem}

The equations of motion for a spacecraft under the influence two massive bodies in a mutually circular orbit can be written in a rotating frame as:

$$
\ddot{\mathbf{r}}+2 \boldsymbol{\Omega} \times \dot{\mathbf{r}}+\boldsymbol{\Omega} \times(\boldsymbol{\Omega} \times \mathbf{r})=\mathbf{a}+\nabla U(\mathbf{r})
$$

where $\mathbf{r}$ is the spacecraft position vector, $\boldsymbol{\Omega}$ is the angular velocity of the rotating frame, $\mathbf{a}$ is an acceleration from a propulsion unit acting on the spacecraft, and $U(\mathbf{r})$ is the three-body problem gravitational potential given by:

$$
U(\mathbf{r})=\frac{1-\mu}{\left|\mathbf{r}_{1}\right|}+\frac{\mu}{\left|\mathbf{r}_{2}\right|}
$$


Here $U(\mathbf{r})$ has been normalized by the the ratio of the masses of the attracting bodies, $\mu=m_{2} /\left(m_{1}+m_{2}\right)$, where $m_{1} \geq m_{2}$ and the sum of the masses is unity. $\mathbf{r}_{1}$ and $\mathbf{r}_{2}$ are the position vectors from the primary bodies to the sail given by:

$$
\begin{aligned}
& \mathbf{r}_{1}=(x+\mu) \mathbf{e}_{x}+y \mathbf{e}_{y}+z \mathbf{e}_{z} \\
& \mathbf{r}_{2}=(x-1+\mu) \mathbf{e}_{x}+y \mathbf{e}_{y}+z \mathbf{e}_{z}
\end{aligned}
$$

where the unit vectors $\mathbf{e}_{x}, \mathbf{e}_{y}, \mathbf{e}_{z}$ denote the $x, y$, and $z$ directions along the rotating coordinate frame.

The system's angular velocity is perpendicular to the plane in which the primaries orbit and is defined to be the z-direction, i.e., $\boldsymbol{\Omega}=\Omega \mathbf{e}_{z}$ as shown in figure 1. Writing the equations of motion in scalar form and normalizing $\Omega=1$, we obtain:

$$
\begin{aligned}
\ddot{x} & =2 \dot{y}+x-\frac{(1-\mu)(x+\mu)}{\left|\mathbf{r}_{1}\right|^{3}}-\frac{\mu}{\left|\mathbf{r}_{2}\right|^{3}}(x+\mu-1)+a_{x} \\
\ddot{y} & =-2 \dot{x}+y-\frac{(1-\mu) y}{\left|\mathbf{r}_{1}\right|^{3}}-\frac{\mu y}{\left|\mathbf{r}_{2}\right|^{3}}+a_{y} \\
\ddot{z} & =-\frac{(1-\mu) z}{\left|\mathbf{r}_{1}\right|^{3}}-\frac{\mu z}{\left|\mathbf{r}_{2}\right|^{3}}+a_{z}
\end{aligned}
$$

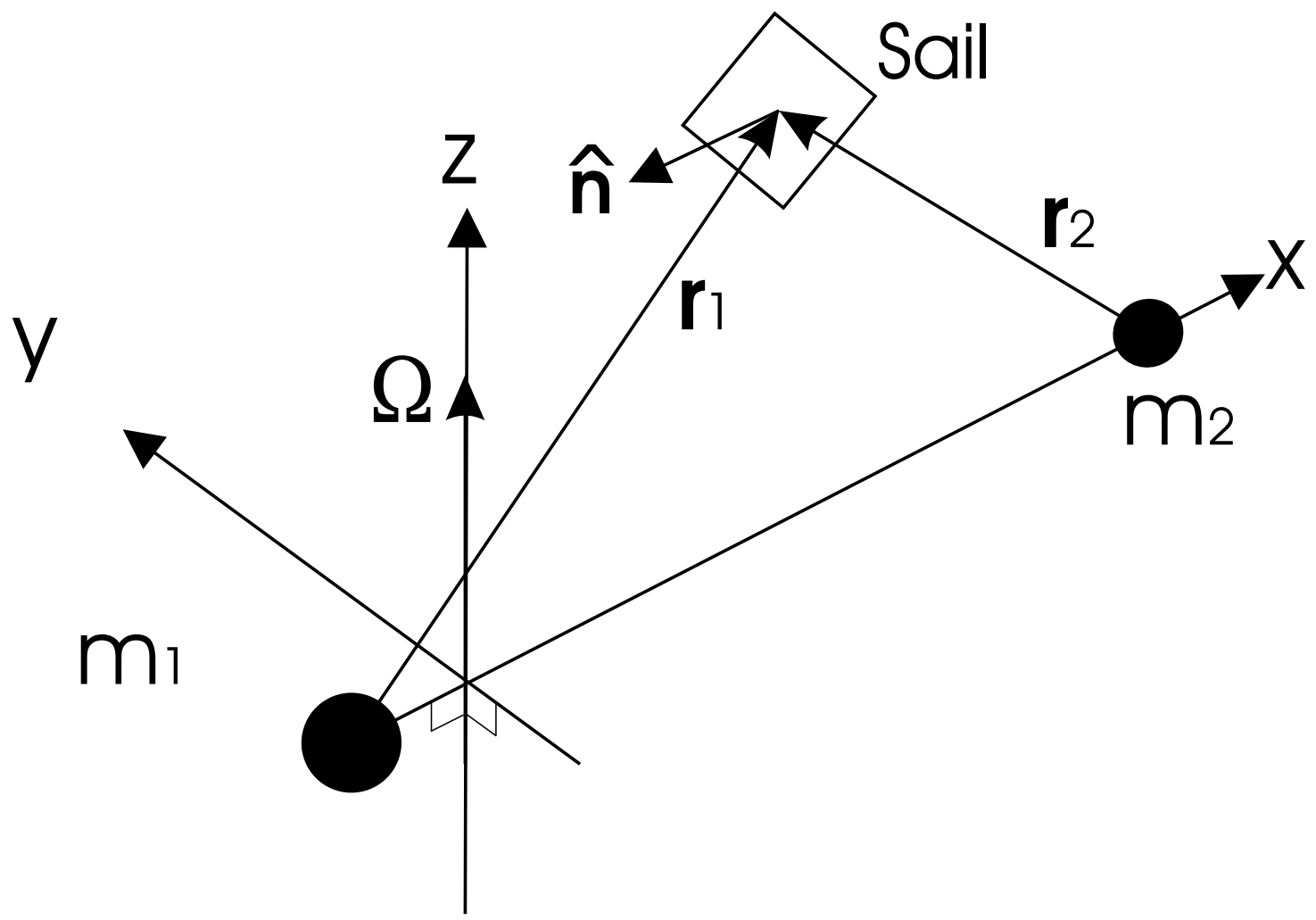

Figure 1. Geometry of the Restricted Three Body Problem.

\section{Sail Propulsive Model}

Our initial studies for this problem are done using an ideal flat sail, although the approach can be generalized to realistic sail models. ${ }^{3}$ Introducing the sail lightness number $\beta,{ }^{4}$ the sail acceleration can be defined in terms of the restricted-three body problem parameters. The sail lightness number $\beta$ is defined as the ratio of the magnitude of the sail acceleration due to the solar radiation pressure and the acceleration caused by 
the sun's gravitational attraction ${ }^{4}$ when the sail is oriented face-on to the sun. Thus, the acceleration due to the propulsion of an ideal sail is given by:

$$
\mathbf{a}=-\frac{\beta(1-\mu)}{\left|\mathbf{r}_{1}\right|^{2}}\left(\mathbf{r}_{1} \cdot \hat{\mathbf{n}}\right)^{2} \hat{\mathbf{n}}
$$

where $\hat{\mathbf{n}}$ is the sail normal vector pointing from the sail surface into the sun's hemisphere. $\hat{\mathbf{n}}$ can be described in terms of the control angles $\alpha$ and $\delta$; sun-sail angle and clock angle, respectively. The control angles are measured with respect to the rotating frame as shown in figure 2 . The acceleration for an ideal sail is in the opposite direction of $\hat{\mathbf{n}}$, i.e. $\mathbf{a}=-a \hat{\mathbf{n}}$ Thus, the acceleration scalar components in terms of the control angles are:

$$
\left[\begin{array}{c}
a_{x} \\
a_{y} \\
a_{z}
\end{array}\right]=\frac{\beta(1-\mu)}{\left|\mathbf{r}_{1}\right|^{2}} \cos ^{2} \alpha\left[\begin{array}{c}
\cos \alpha \\
-\sin \alpha \cos \delta \\
-\sin \alpha \sin \delta
\end{array}\right]
$$

Note that the acceleration is maximum when $\hat{\mathbf{n}}$ is antiparallel to $\mathbf{r}_{1}$.

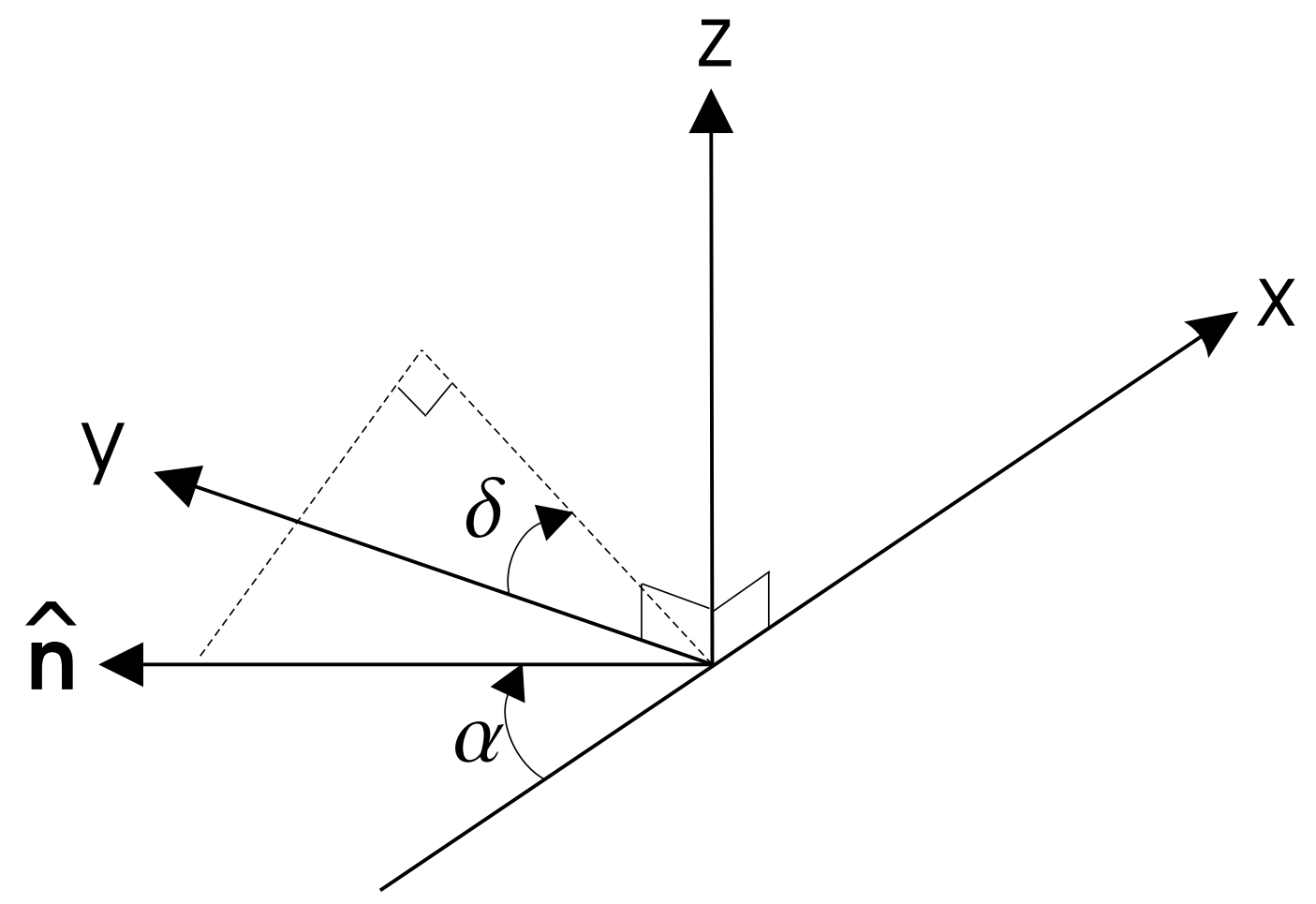

Figure 2. Sail Control Angles. $\alpha$ is the angle between $\hat{\mathbf{n}}$ and the $-\mathrm{x}$ axis, $\delta$ is the angle between the projection of $\hat{n}$ into the $(y, z)$-plane and the $y$-axis.

\section{Sub- $L_{1}$ Points}

Solar sails allow a sailcraft to create artificial Lagrange points ${ }^{4}$ so called sub- $L_{1}$ points. $^{2}$ These new locations have a number of applications since they are closer to the sun than the $L_{1}$ point. The location of a new sub- $L_{1}$ point is dependent on the sail lightness number $\beta$. In order to find a sub- $L_{1}$ point with the sail plane perpendicular to the solar flux, we need to write the equations of motion along the radial direction using the CR3BP: 


$$
\ddot{x}=x-\frac{\mu(x-1+\mu)}{|(x-1+\mu)|^{3}}-\frac{(1-\mu)(x+\mu)}{|(x+\mu)|^{3}}+a_{x}
$$

where $a_{x}$ is the force along the radial direction which for $\alpha=0$ is given by:

$$
a_{x}=\beta \frac{1-\mu}{|(x+\mu)|^{2}}
$$

Setting Eq. (10) equal to zero, we can solve for the lightness number that would yield an equilibrium point in terms of $\mu$ and for a specific $x=x_{L_{1}}$ :

$$
\beta=\frac{\left|\left(x_{L_{1}}+\mu\right)\right|^{2}}{1-\mu}\left[-x_{L_{1}}+\frac{\mu\left(x_{L_{1}}-1+\mu\right)}{\left|\left(x_{L_{1}}-1+\mu\right)\right|^{3}}+\frac{(1-\mu)\left(x_{L_{1}}+\mu\right)}{\left|\left(x_{L_{1}}+\mu\right)\right|^{3}}\right]
$$

\section{Circular Restricted Three Body Problem in Cylindrical Coordinates}

If the sail produces a higher force than originally expected then it cannot be stationed at the sub- $L_{1}$ point. One solution is to recompute a new sub- $L_{1}$ point $x_{L_{1}}^{\prime}<x_{L_{1}}$, however, this approach may force the sail to fly in a regime that it was not designed for. Another possibility is to used the extra force to orbit the sub- $L_{1}$ point. The second option provides a better approach since it allows the sail to maintain the required $x_{L_{1}}$ location.

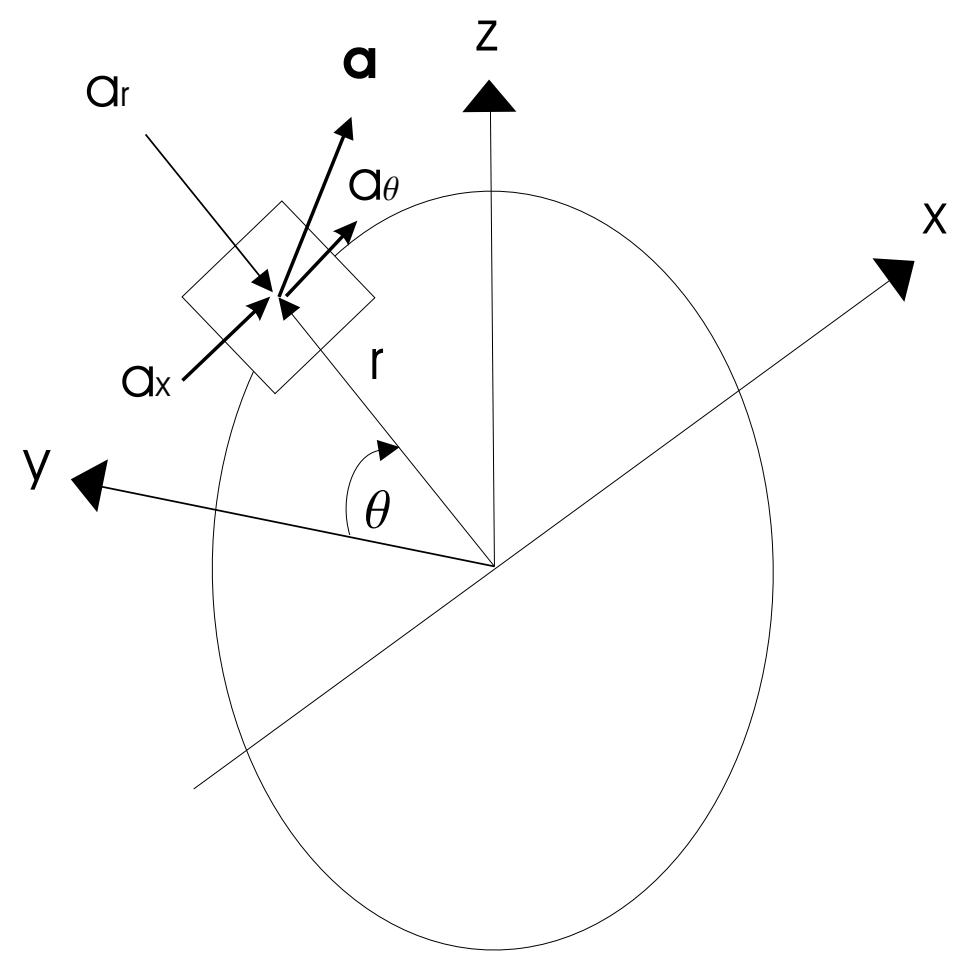

Figure 3. Sail in Orbit about Sub- $L_{1}$ Point.

For orbiting the sub- $L_{1}$ point it is helpful to write the equations of motion in a cylindrical coordinate frame rotating about the nominal equilibrium as shown in figure 3. This describes a cylindrical coordinate frame with its axis of symmetry along the x-axis. Let $y=r \cos \theta$ and $z=r \sin \theta$. The kinematics of the $y$ and $z$ coordinates in the new variables is:

$$
\dot{y}=\dot{r} \cos \theta-r \dot{\theta} \sin \theta
$$




$$
\begin{aligned}
\dot{z} & =\dot{r} \sin \theta+r \dot{\theta} \cos \theta \\
\ddot{y} & =\ddot{r} \cos \theta-2 \dot{r} \dot{\theta} \sin \theta-r \dot{\theta}^{2} \cos \theta-r \ddot{\theta} \sin \theta \\
\ddot{z} & =\ddot{r} \sin \theta+2 \dot{r} \dot{\theta} \cos \theta-r \dot{\theta}^{2} \sin \theta+r \ddot{\theta} \cos \theta
\end{aligned}
$$

Eqs. (6) - (7) become

$$
\begin{aligned}
& \ddot{y}=-2 \dot{x}+\left(1-\frac{1-\mu}{\left|\mathbf{r}_{1}\right|^{3}}-\frac{\mu}{\left|\mathbf{r}_{2}\right|^{3}}\right) r \cos \theta+a_{y} \\
& \ddot{z}=-\left(\frac{1-\mu}{\left|\mathbf{r}_{1}\right|^{3}}+\frac{\mu}{\left|\mathbf{r}_{2}\right|^{3}}\right) r \sin \theta+a_{z}
\end{aligned}
$$

Substituting Eqs. (15) and (16) into the above equations:

$$
\left[\begin{array}{cc}
\cos \theta & -r \sin \theta \\
\sin \theta & r \cos \theta
\end{array}\right]\left[\begin{array}{c}
\ddot{r} \\
\ddot{\theta}
\end{array}\right]=\left[\begin{array}{c}
-2 \dot{x}+\left(1-\frac{1-\mu}{\left|\mathbf{r}_{1}\right|^{3}}-\frac{\mu}{\left|\mathbf{r}_{2}\right|^{3}}\right) r \cos \theta+2 \dot{r} \dot{\theta} \sin \theta+r \dot{\theta}^{2} \cos \theta \\
-\left(\frac{(1-\mu)}{\left|\mathbf{r}_{1}\right|^{3}}+\frac{\mu}{\left|\mathbf{r}_{2}\right|^{3}}\right) r \sin \theta-2 \dot{r} \dot{\theta} \cos \theta+r \dot{\theta}^{2} \sin \theta
\end{array}\right]
$$

solving for $\ddot{r}$ and $\ddot{\theta}$ the equations of motion in the new coordinate frame are:

$$
\begin{aligned}
\ddot{x} & =2 \dot{r} \cos \theta-2 r \dot{\theta} \sin \theta+x-\frac{(1-\mu)(x+\mu)}{\left|\mathbf{r}_{1}\right|^{3}}-\frac{\mu}{\left|\mathbf{r}_{2}\right|^{3}}(x+\mu-1)+a_{x} \\
\ddot{r} & =-2 \dot{x} \cos \theta+r \cos ^{2} \theta-\left(\frac{1-\mu}{\left|\mathbf{r}_{1}\right|^{3}}+\frac{\mu}{\left|\mathbf{r}_{2}\right|^{3}}\right) r+r \dot{\theta}^{2}+a_{r} \\
\ddot{\theta} & =2 \dot{x} \frac{\sin \theta}{r}-\sin \theta \cos \theta-2 \frac{\dot{r} \dot{\theta}}{r}+\frac{a_{\theta}}{r}
\end{aligned}
$$

where the acceleration in cylindrical coordinates is given by:

$$
\left[\begin{array}{l}
a_{x} \\
a_{r} \\
a_{\theta}
\end{array}\right]=\frac{\beta(1-\mu)}{\left|\mathbf{r}_{1}\right|^{2}} \cos ^{2} \alpha\left[\begin{array}{c}
\cos \alpha \\
-\sin \alpha \cos (\delta-\theta) \\
-\sin \alpha \sin (\delta-\theta)
\end{array}\right]
$$

\section{Excess Performance in the Sail Propulsion Unit}

The derivation of the above equilibrium point was performed assuming exact knowledge of the propulsion force of the sail. Uncertainties present in the force sail model will change the location of this equilibrium point due to either insufficient or excess force available. If the sail resultant force is less that the required force, then it would be impossible to stay at the required equilibrium and a recalculation would be necessary. If the force is more than expected, then through some maneuvering it is still possible to stay at the required equilibrium. In the following we assume that it is desired to stay at the original, designed equilibrium point.

Assume that the actual force created by the sail is larger than the force expected. To maintain the sail at the same fixed sub- $L_{1}$ point, one would have to orient the sail continuously in order to shed the excess force. There several ways of doing this, for example, the orientation of the sail can be dithered by oscillating the sail in order to keep the average acceleration equal to the required acceleration:

$$
\overline{\mathbf{a}}=\frac{1}{T} \int_{0}^{T} \mathbf{a}(t) d t=\left[\begin{array}{c}
a_{x_{L_{1}}} \\
0 \\
0
\end{array}\right]
$$

where $a_{x_{L_{1}}}$ is the nominal acceleration to station the sail at the equilibrium.

Another option is to use the excess force to orbit the equilibrium. This can be done by continuously steering the sail in order to cancel the centrifugal forces with the excess force. The sun-sail angle, not 
necessarily constant, must be such that the average force along the radial direction is equal to the original nominal force. Assume that the actual acceleration exceeds the nominal acceleration by a constant $k$, so that the actual force along the $\mathrm{x}$-direction is:

$$
a_{x}=k \beta \frac{1-\mu}{x+\mu}=\beta^{\prime} \frac{1-\mu}{x+\mu}
$$

where $\beta^{\prime}$ is the actual sail lightness number.

Since this acceleration is larger than required, the sail must be oriented with a non-zero $\alpha$ with the constraint that the force along the x-direction must be equal to the nominal acceleration. With this in mind, the following relation needs to be satisfied:

$$
\beta \frac{1-\mu}{x+\mu}=\beta^{\prime} \frac{1-\mu}{x+\mu} \cos ^{3} \alpha
$$

which provides the required sun-sail angle:

$$
\alpha_{0}=\cos ^{-1}\left(\frac{1}{k^{1 / 3}}\right)
$$

Certainly, if the sail is maintained at this constant attitude there will be a force generated orthogonal to the $x$-axis which will drive the sail away from the equilibrium. However, if the sail is to orbit the equilibrium in a quasi-circular orbit, then the excess force can be used to cancel the centrifugal acceleration by directing it toward the equilibrium. Ideally, for accomplishing this, $\delta-\theta$ must be set equal to zero in Eq. (23). Then, the acceleration due to the solar radiation pressure along the radial direction is:

$$
a_{r}=-\frac{\beta^{\prime}(1-\mu)}{r_{1}^{2}} \cos ^{2} \alpha_{0} \sin \alpha_{0}
$$

The radial acceleration must balance the centrifugal acceleration in the orbit, thus $a_{r}=-a_{c}$. Given this available acceleration it is now possible to design the orbit the sail has to follow. Assume that a radius $r_{d}$ is desired, then the velocity $v_{d}$ and angular velocity $\theta_{d}$ needed to stay in a circular orbit are:

$$
\begin{aligned}
& v_{d}=\sqrt{a_{c} r_{d}} \\
& \dot{\theta}_{d}=\sqrt{\frac{a_{c}}{r_{d}}}
\end{aligned}
$$

Choosing values of $r_{d}=1 \times 10^{-5}$, in the normalized system, $k=1.1$, and $\alpha$ and $v_{c}$ determined from Eqs. (27) and (29), respectively, we can apply this concept to a simulation. The chosen initial conditions are $\mathbf{r}_{0}=\left[x_{L_{1}}, r_{d}, 0\right]$, where $x_{0}$ is the location of the sub- $L_{1}$ point, and $\dot{\mathbf{r}}=\left[0,0, v_{c}\right]$. The simulated free dynamics are shown in Fig. 4. This orbit is unstable and slides away from the equilibrium point. This is expected as the equilibrium point is itself unstable.

\section{Control of Sail Orbit about a Sub- $L_{1}$ Point}

From the equations of motion, the sail control angles $\alpha$ and $\delta$ allow for the control of the sail about the equilibrium point. The sail position about the $x$-axis is affected by $\alpha$ and the $r$ and $\theta$ directions by both $\alpha$ and $\delta$.

The $x$-position is maintained at $x_{L_{1}}$ by adjusting $\alpha$ in such a way that it will cancel disturbances that drive the sail away form $x_{L_{1}}$. This $\alpha$ can be chosen from a linearized controller or a proportional-derivative controller developed in this section.

Once $\alpha$ is chosen, $\delta$ is left to control the $r$ and $\theta$ directions. The objective here is to keep the sail in a circular or semicircular orbit by driving $\dot{r}$ and $\ddot{r}$ to zero (or close to zero). The approach taken here is to design an orbit with a fixed radius and use energy as a reference for feedback in the control law. 


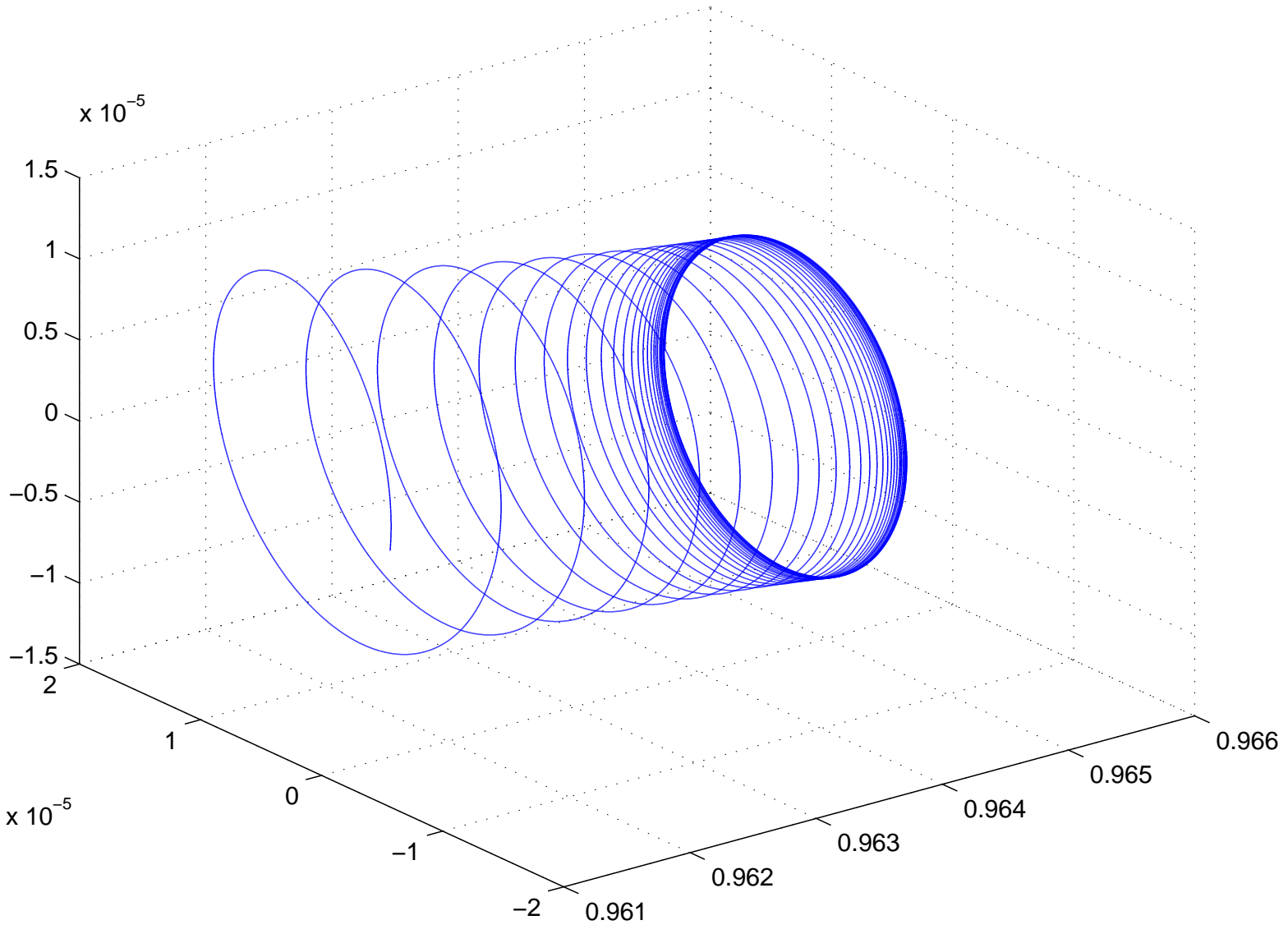

Figure 4. Long Term Orbit About sub- $L_{1}$ Point for Uncontrolled Dynamics.

\section{A. Control of Sail $x$-Position}

A feedback linear controller is developed by linearizing the $x$-dynamics about $x_{L_{1}}$ and $\alpha_{0}$. This controller is then applied to the non-linear system. From Eq. (5), $\ddot{x}=f(\mathbf{x})$, the linearized equations about the equilibrium are:

$$
\left[\begin{array}{c}
\delta \dot{x} \\
\delta \ddot{x}
\end{array}\right]=\left[\begin{array}{ll}
0 & 1 \\
b & 0
\end{array}\right]\left[\begin{array}{l}
\delta x \\
\delta \dot{x}
\end{array}\right]+\left[\begin{array}{l}
0 \\
c
\end{array}\right] \delta \alpha
$$

where

$$
\begin{aligned}
b & =\left.\frac{\partial a_{x}}{\partial x}\right|_{L_{1}}+\left.\frac{\partial f(\mathbf{x})}{\partial x}\right|_{L_{1}} \\
\left.\frac{\partial a_{x}}{\partial x}\right|_{L_{1}} & =-2 \frac{1-\mu}{\left|x_{L_{1}}+\mu\right|^{3}} \cos ^{3} \alpha_{0} \\
\left.\frac{\partial f(\mathbf{x})}{\partial x}\right|_{L_{1}} & =1+2 \frac{1-\mu}{\left|x_{L_{1}}+\mu\right|^{3}}+2 \frac{\mu}{\left|x_{L_{1}}+\mu-1\right|^{3}} \\
c & =\left.\frac{\partial a_{x}}{\partial \alpha}\right|_{L_{1}}=-3 \frac{1-\mu}{\left|x_{L_{1}}+\mu\right|^{2}} \cos ^{2} \alpha_{0} \sin \alpha_{0}
\end{aligned}
$$

Using simple controllability tests we see that the system is controllable. Now it is possible to design the linear feedback controller by letting: 


$$
\delta \alpha=\left[\begin{array}{ll}
k_{1} & k_{2}
\end{array}\right]\left[\begin{array}{l}
\delta x \\
\delta \dot{x}
\end{array}\right]
$$

The closed-loop system becomes:

$$
\left[\begin{array}{l}
\delta \dot{x} \\
\delta \ddot{x}
\end{array}\right]=\left[\begin{array}{cc}
0 & 1 \\
b+k_{1} c & k_{2} c
\end{array}\right]\left[\begin{array}{c}
\delta x \\
\delta \dot{x}
\end{array}\right]
$$

with characteristic polynomial:

$$
p(\lambda)=\lambda^{2}-\lambda\left(k_{2} c\right)-c k_{1}-b=0
$$

for stability we want the coefficients to be positive. Note that $b>0$, and $c<0$. Then the gains have to satisfy:

$$
\begin{aligned}
& k_{2}>0 \\
& k_{1}>\frac{b}{c}
\end{aligned}
$$

A simulation using the linear feedback controller was performed using gains $k_{1}=7000$ and $k_{2}=200$. Figure 5 shows the simulation results. The sail was initially located sunward of the sub- $L_{1}$ point at a distance of $2 \times 10^{-5}$ in normalized units. The controller stabilizes the sail around the equilibrium, but is not asymptotically stable since the control corrections are done on an average value of $\alpha$ and the linearized dynamics do not take into account the coupling with the $r$ and $\theta$ variables, however the sail is kept within some $\epsilon$ of the equilibrium.

Another approach we consider is to apply a proportional-derivative controller to the position deviation and the rate at which the sailcraft is receding from the sub- $L_{1}$ point. Let's suppose that the current sail position is $x$ and the sub- $L_{1}$ point is at $x_{L_{1}}$, then if $x>x_{L_{1}}$ the angle $\alpha$ must be increased to reduce the force along the $x$-axis and move sail in the direction of the sun. If $x<x_{L_{1}}$ the opposite should be done. Now if the sail is already at the required equilibrium and $\dot{x}>0$, then $\alpha$ must be increased, and if $\dot{x}<0$, then $\alpha$ must be decreased. Then the controller will have the form $\delta \alpha=c_{1}\left(x-x_{L_{1}}\right)+c_{2} \dot{x}$, where $c_{1}$ and $c_{2}$ are the PD-controller constant gains.

The PD-controller will help bring the sail to the sub- $L_{1}$ point. Once the sail is there, it will experience accelerations away from the equilibrium due to the coupling of the dynamics. To reject these disturbances $\alpha$ needs to be corrected at every instant in the orbit to account for the acceleration arising from the $r$ and $\theta$ coordinates. This can be done by finding an $\alpha$ that yields a zero net acceleration in the $x$-direction. From Eq. (20), it is possible to find this $\alpha$ by solving:

$$
\cos ^{3} \alpha_{1}=-\left[2 \dot{r} \cos \theta-2 r \dot{\theta} \sin \theta+x_{L_{1}}-\frac{(1-\mu)\left(x_{L_{1}}+\mu\right)}{\left|\mathbf{r}_{1}\left(x_{L_{1}}\right)\right|^{3}}-\frac{\mu}{\left|\mathbf{r}_{2}\left(x_{L_{1}}\right)\right|^{3}}\left(x_{L_{1}}+\mu-1\right)\right] \frac{\left|\mathbf{r}_{1}\left(x_{L_{1}}\right)\right|^{2}}{\beta^{\prime}(1-\mu)}(41)
$$

Even though this equation is cubic, $\cos ^{3} x$ is one-to-one in the domain of alpha, 0 to $\pi / 2$, thus there will always be a real solution. The active PD-control law that stabilizes the sail in the $x$-direction about the sub- $L_{1}$ point is then:

$$
\alpha=\alpha_{1}+c_{1}\left(x-x_{0}\right)+c_{2} \dot{x}
$$

where $\alpha_{1}$ is the real solution of Eq. (41).

Figure 6 shows the sail's trajectory and $x$-position using this controller with $c_{1}=3000$ and $c_{2}=500$ with the sail starting at a distance of $2 \times 10^{-5}$, in the normalized units, sunward of the sub- $L_{1}$ point.

In order to maintain the sail exactly at $x_{L_{1}}, \alpha$ has to be adjusted at every point in the orbit. Continuous active modulation is necessary to avoid oscillations in the $x$-direction and may not always be feasible. 


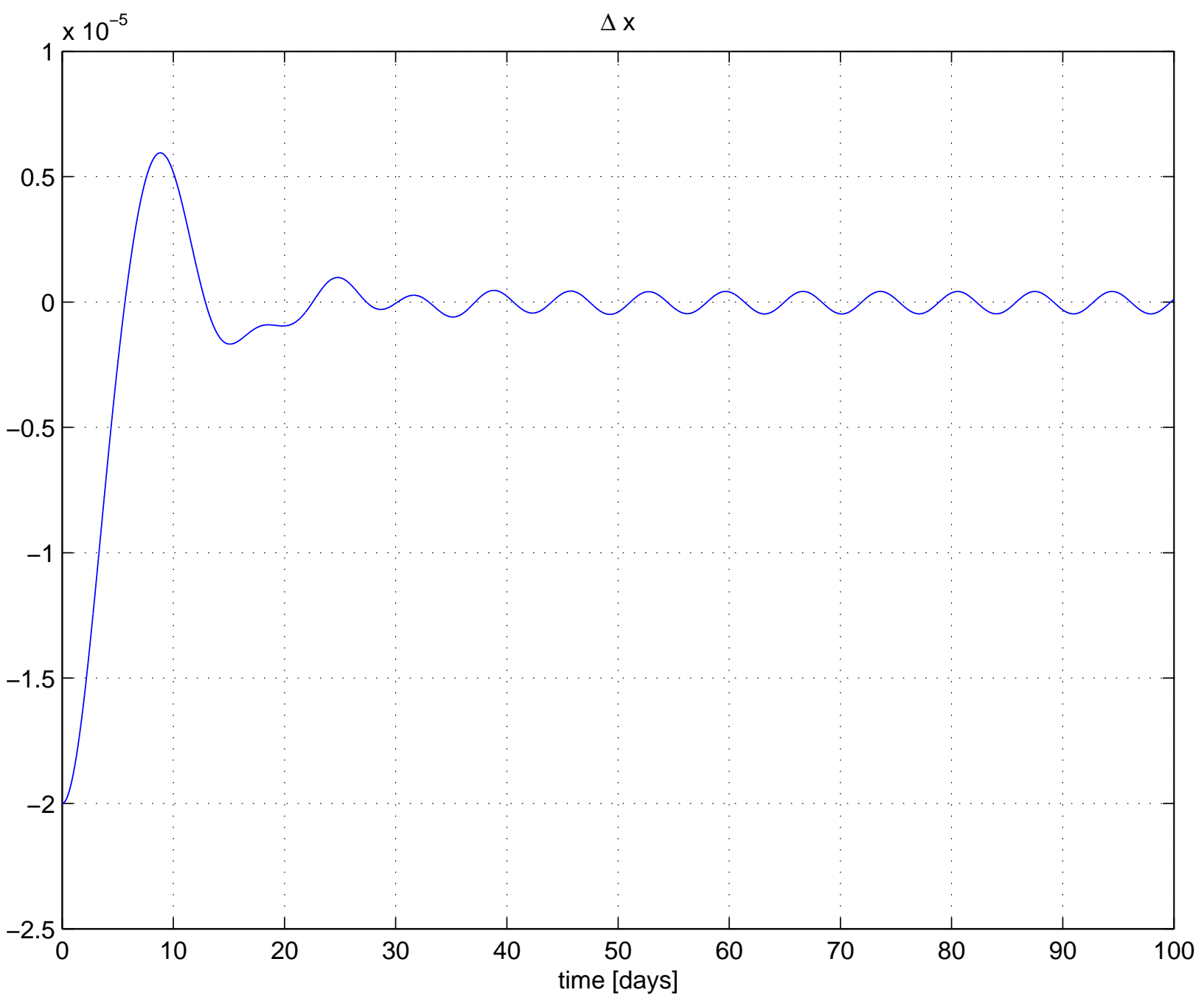

Figure 5. Linear Feedback Control on Sail x-Position.

Although a completely constant sun-sail angle is not practical, since the controller needs to correct from deviations, the part that is dependent on the orbit position can be neglected on the controller. Thus, the controller still is given by Eq. (42) with $\alpha_{1}$ satisfying:

$$
\cos ^{3} \alpha_{1}=-\left[x_{L_{1}}-\frac{(1-\mu)\left(x_{L_{1}}+\mu\right)}{\left|\mathbf{r}_{1}\left(x_{L_{1}}\right)\right|^{3}}-\frac{\mu}{\left|\mathbf{r}_{2}\left(x_{L_{1}}\right)\right|^{3}}\left(x_{L_{1}}+\mu-1\right)\right] \frac{\left|\mathbf{r}_{1}\left(x_{L_{1}}\right)\right|^{2}}{\beta^{\prime}(1-\mu)}
$$

Notice that $\alpha_{1}$ is constant for this case and the new controller has a similar form and performance as the linear feedback controller. Hence, it is expected that the sail $x$-position oscillates about $x_{L_{1}}$. The amplitude of the oscillations are dependent on the PD gains.

\section{B. Control of Sail Orbit Radius}

Once the sail is stabilized at the equilibrium, $\dot{x}$ becomes zero (or sufficiently small) and consequently the dynamics in the $r$ and $\theta$ coordinates decouple from the varying $x$ coordinate. The dynamical equations governing this motion reduce to:

$$
\ddot{r}=r \cos ^{2} \theta-\left(\frac{1-\mu}{\left|\mathbf{r}_{1}\right|^{3}}+\frac{\mu}{\left|\mathbf{r}_{2}\right|^{3}}\right) r+r \dot{\theta}^{2}-\frac{\beta(1-\mu)}{\left|\mathbf{r}_{1}\right|^{2}} \cos ^{2} \alpha \sin \alpha \cos \bar{\delta}
$$

10 of 18 

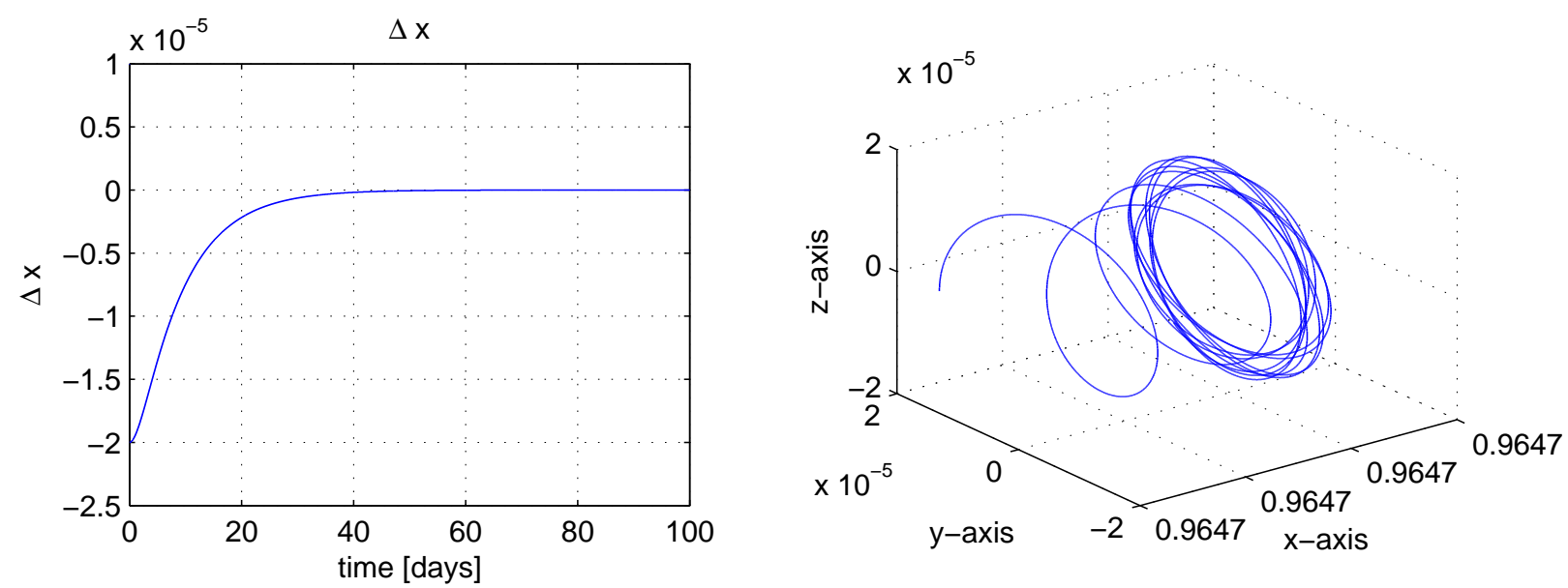

Figure 6. Active Proportional Derivative Control on Sail $x$-Position.

$$
\ddot{\theta}=-\sin \theta \cos \theta-2 \frac{\dot{r} \dot{\theta}}{r}-\frac{1}{r} \frac{\beta(1-\mu)}{\left|\mathbf{r}_{1}\right|^{2}} \cos ^{2} \alpha \sin \alpha \sin \bar{\delta}
$$

where the new control variable is just $\bar{\delta}=\delta-\theta$. Another controller is needed to maintain the sail at the required orbit radius. The sail radius about the sub- $L_{1}$ point can differ from the nominal radius if deviations in the orbit insertion arise, and can be quite large. In figure 6 we can see the excursion in the radius $r$ for the above example. Rather than controlling the orbit through $r$ and $\theta$ directly, a simpler control law can be defined if the effective kinetic energy about the sub- $L_{1}$ point of the orbit is used.

The sail kinetic energy about the sub- $L_{1}$ point is given by:

$$
E=\frac{1}{2}\left(r^{2} \dot{\theta}^{2}+\dot{r}^{2}\right)
$$

It is desired to put a sail in a orbit with radius $r_{d}$. This orbit has an energy $E_{0}=1 / 2 r_{d}^{2} \dot{\theta}_{d}^{2}$, which is called the reference energy. The error between the actual and reference kinetic energy about the sub- $L_{1}$ point orbit, which can be used to track the deviation from the actual to the desired sail orbit, is $\tilde{E}=E-E_{0}$. The sail orbit is controlled by defining a proportional controller of the form:

$$
\bar{\delta}=-c_{e} \sin ^{-1} \frac{\tilde{E}}{r_{d}}
$$

where $c_{e}$ is a constant gain and $r_{d}$ is used as a scaling factor. Note that $E_{0}$ is not constant but is a function of $\alpha$ :

$$
E_{0}=\frac{1}{2} r_{d}^{2} \dot{\theta}^{2}=\frac{1}{2} r_{d}\left|a_{t}\right|=\frac{1}{2} r_{d} \frac{\beta^{\prime}(1-\mu)}{\left|\mathbf{r}_{1}\right|^{2}} \cos \alpha \sin \alpha
$$

Figs. 7 shows the results of using this controller with $c_{e}=10$. The sail was started at a radius $30 \%$ larger that the nominal and sunward to the sub- $L_{1}$ point. It can be seen that the controller is able to track the nominal energy. However, since the nominal energy is not constant, the sail orbit oscillates about the nominal radius with very small deviations. In the same figure, the steady state sail orbit is shown to be a closed loop.

\section{Sail Propulsive Model Estimation}

Our whole approach is predicated on the possibility that the sail propulsive model being used in the sail controller differs from the some ideal propulsive model. However, initially only an estimation of the 

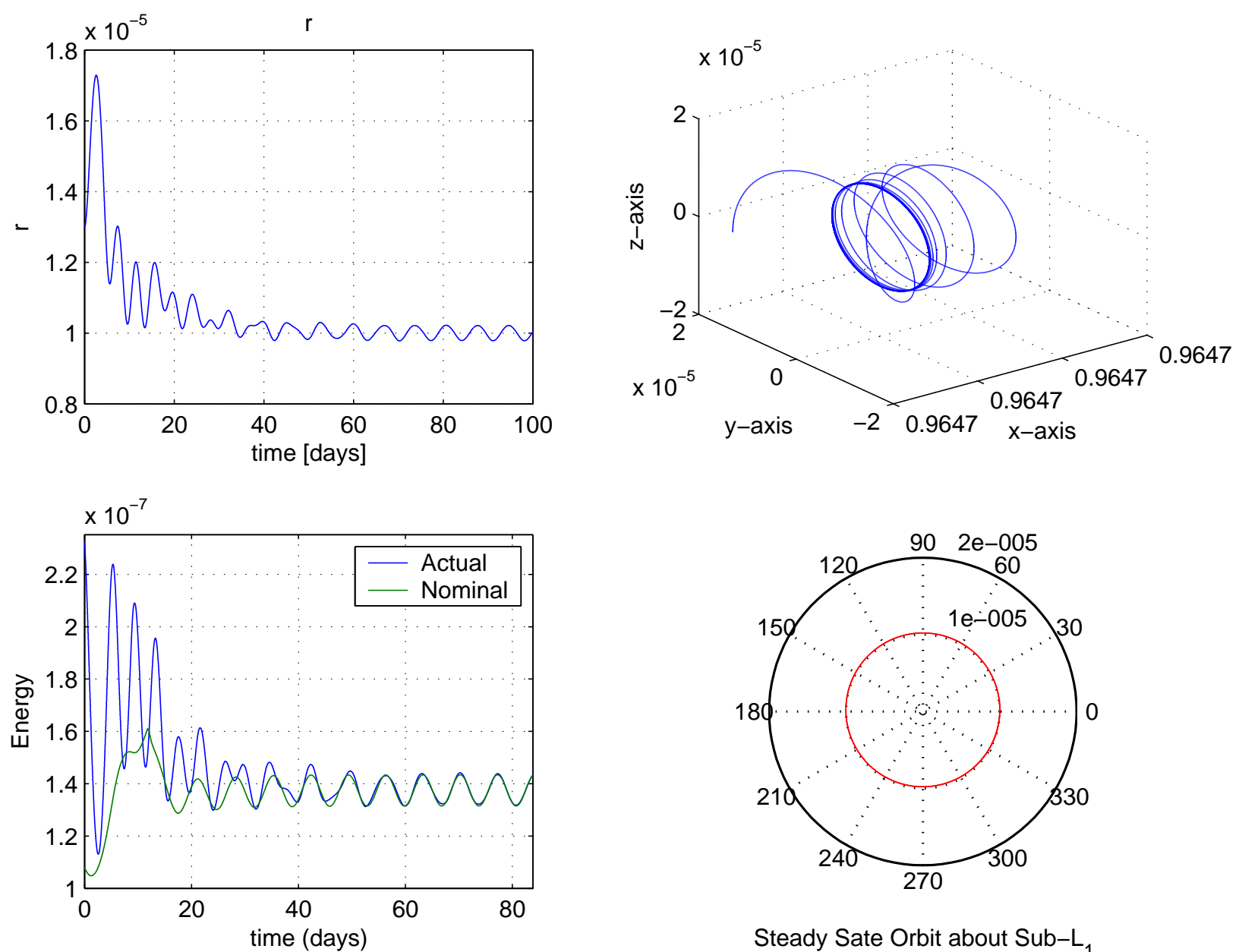

Figure 7. Energy Controlled Sail.

propulsive sail model may be known rather than the actual propulsive model. If the estimated propulsive model is not too far off from the actual model, then the controller will still work, but it may not settle into the required position but shift into a new equilibrium instead. Specifically, the $x$-position will be affected by the uncertainty in the propulsive model. Using the difference between the new equilibrium and the required sub- $L_{1}$ point it is possible to refine the estimate of the propulsive model. For achieving this a formalism for updating the propulsive parameters can be obtained from the control laws. The objective is to find the sensitivity of changes in the $x$-equilibrium with respect to changes in the propulsive model, which for the ideal sail is the $k$ parameter. This sensitivity can be written as:

$$
\frac{\partial x}{\partial k}=\frac{\partial x}{\partial \alpha} \frac{\partial \alpha}{\partial k}
$$

each of the partial derivatives in the right-hand side of the equation can be computed from the control laws, Eqs. (42)-(43). Note that $\alpha$ is time varying, nonetheless, an average value can be used to approximate the partial derivative. Thus, from the average value of $\alpha$ in Eq. (43), the partial derivative of $\bar{\alpha}$ with respect to $k$ is

$$
\frac{\partial \bar{\alpha}}{\partial k}=-\left[x_{L_{1}}-\frac{(1-\mu)\left(x_{L_{1}}+\mu\right)}{\left|\mathbf{r}_{1}\left(x_{L_{1}}\right)\right|^{3}} \frac{\mu}{\left|\mathbf{r}_{2}\left(x_{L_{1}}\right)\right|^{3}}\left(x_{L_{1}}+\mu-1\right)\right] \frac{\left|\mathbf{r}_{1}\left(x_{L_{1}}\right)\right|^{2}}{\beta(1-\mu)} \cdot \frac{1}{k^{2} 3 \cos ^{2} \bar{\alpha} \sin \bar{\alpha}}
$$


the other partial derivative, obtained from Eq. (42), is simply

$$
\frac{\partial x}{\partial \bar{\alpha}}=\frac{1}{c_{1}}
$$

Thus, once the sail settles into a constant or nearly constant distance from the sun along the $x$-direction, the difference between the current and desired position can be used to update $k$ through:

$$
\Delta k \approx \frac{\Delta x}{\partial x / \partial k}
$$

Several iterations are in general necessary before converging into the the actual $k$. This current approach shows the feasibility of an estimation method and is not optimal in general. Future research will focus on an estimation formalism using direct measurements of accelerations and fitting of precise navigation tracking with motion.

Figure 8 shows the application of this idea to two cases. In the first case the controller assumes a $k=1.1$ while the actual is 1.05 and the sail is starts behind the sub- $L_{1}$ point at a distance of $2 \times 10^{-5}$, in the normalized units. In the second case the actual $k$ is 1.15 and the controller is initialized with $k=1.1$ with the sail initially located at the same distance as the first case but sunward of the sub- $L_{1}$ point. In both of these cases, the controller tries to stabilize the sail $x$-position. When the controller senses that it is converging to a different $x$-location, it uses this information to update $k$. After 120 days, $k=1.0493$ and $k=1.1506$ for the first and second cases, respectively.

The estimation can also be done with the second PD-controller presented and shown by the simulation in figure 9. The same two above cases for the implementation of the adaptive controller were used. The sail was placed at distance of $2 \times 10^{-5}$, in the normalized units, sunward and behind of $x_{L_{1}}$. During the simulation, the $k$ parameter is estimated to a value close to the actual, however, since the sail $x$-position does not settle into a constant value, the estimation is not perfect and the $k$ parameter does not converge exactly into the actual value. Despite this, the sail is driven close to the desired location with this control implementation. Once in the vicinity of the sub- $L_{1}$ point the estimator can be turned off.

\section{Sail Control Under Degradation}

The sail membrane will suffer from degradation due to solar radiation and the space environment. ${ }^{1}$ The membrane degradation will result in a loss of propulsion forces acting on the sail. In order to maintain the sail orbiting the sub- $L_{1}$ point this effect must be accounted for in $E_{0}$. It is possible to keep a constant radius about the equilibrium, however, this control law would fail when the force acting on the sail becomes insufficient for maintaining the $x$-position of the equilibrium. A better approach is to make the radius decrease as the sail degrades. Hence, the sail will eventually settle into the sub- $L_{1}$ point when the sail performance degrades to its minimum at $\beta^{\prime}=\beta$. Then, the required energy satisfies:

$$
E_{0}=\frac{1}{2} r_{d}^{2} \dot{\theta}^{2}=\frac{1}{2} r_{d 0}^{2} k^{2} \dot{\theta}^{2}
$$

where $k$ is a function of time, and the orbit radius satisfies $r_{d}(k(0))=r_{d o}$ (the initial value of the orbit radius) and $r_{d}(1)=0$. It is possible to choose how $r_{d}$ changes with degradation. For instance, if $r_{d}$ varies linearly with $k$, then:

$$
E_{0}=\frac{1}{2}\left(\frac{k(t)-1}{k(0)-1}\right)^{2} r_{d 0}^{2} \dot{\theta}^{2}
$$

where $k(t)$ is the value of $k$ at time $\mathrm{t}$, and $k(0)$ is the initial value of $k$. When $k=1$, the sail radius is zero and the sail is at the sub- $L_{1}$ point at which the reference energy is zero. When $k(t)<1$, the sail force is not sufficient to keep it at the equilibrium and a mission redesign must occur. Figure 10 shows a simulation of a solar sail under degradation. The sail was initially positioned sunward of the equilibrium. The controller stabilized the sail at the required $x$-position and the radius was decreased as the sail surface degraded. 

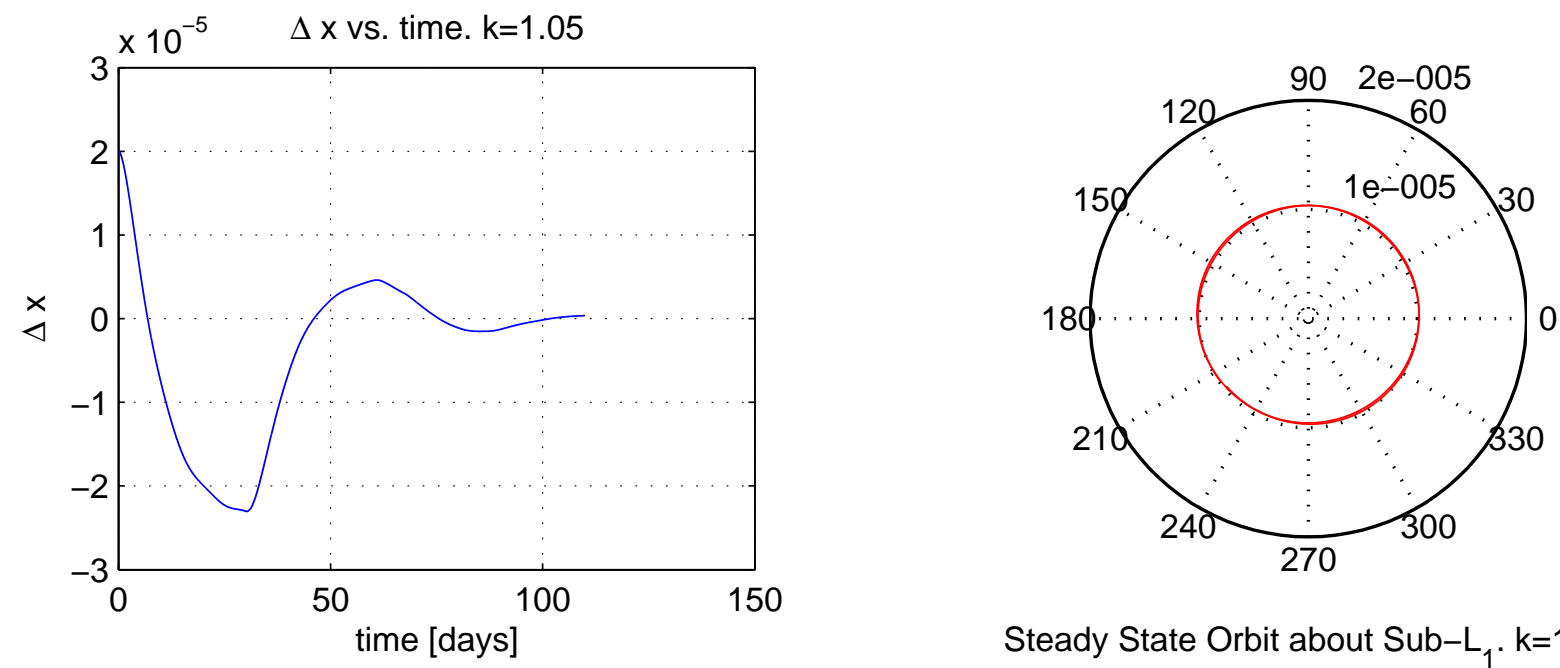

Steady State Orbit about Sub- $\mathrm{L}_{1} \cdot \mathrm{k}=1.05$
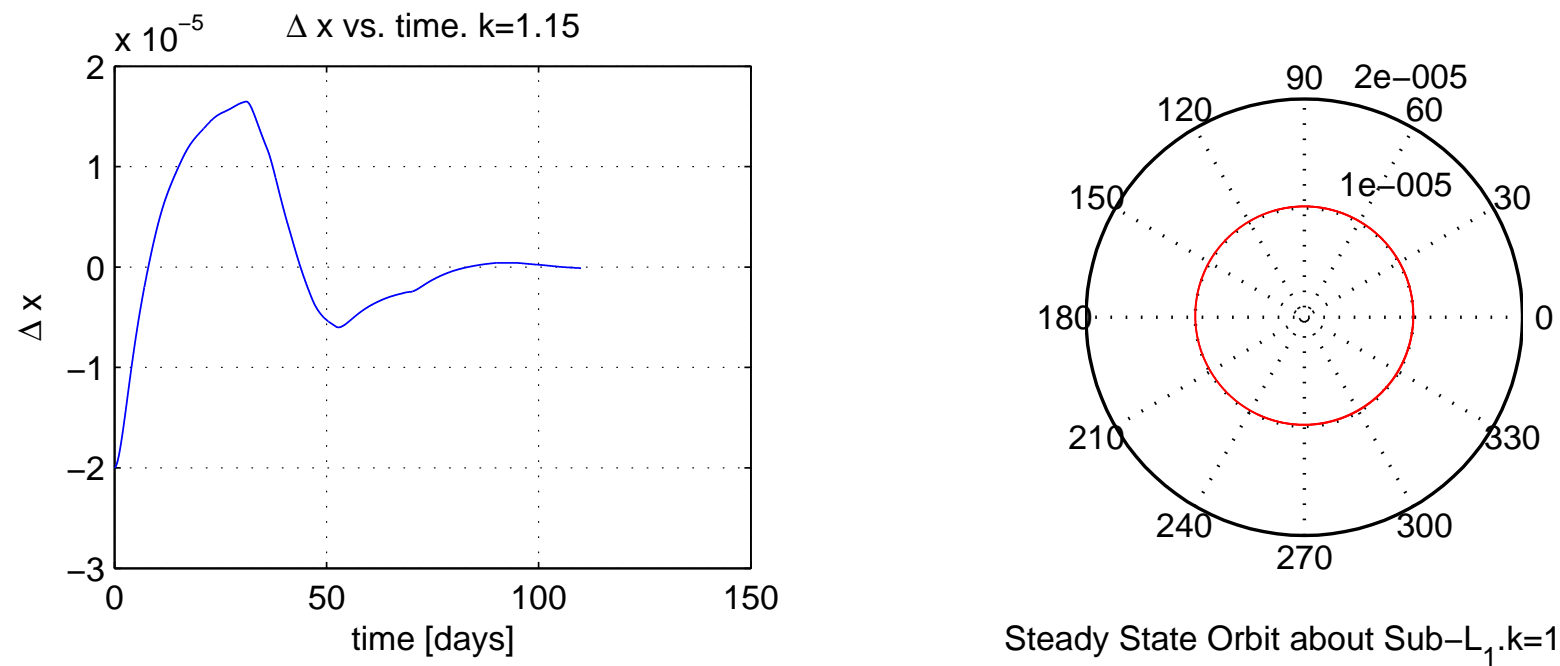

Steady State Orbit about Sub- $\mathrm{L}_{1} \cdot \mathrm{k}=1.15$

Figure 8. Adaptive Controller.

A final test is now given which involves simultaneous control, estimation, and degradation of the sail. Here the sail is considered to undergo surface degradation and at the same time we try to estimate its value to be used in the controller. Initially, the sail is located $2 \times 10^{-5}$ distance units sunward of the equilibrium and with a larger radius than the nominal. For simulation purposes, the sail degrades linearly with time. Initially, the actual value of $\beta^{\prime}$ is 0.115 or $k(0)=1.15$. The initial estimate of $k$ used in the controller is 1.1. After 150 days $k$ decays to 1.0897 while the estimated value is 1.092 . Initially the sail overshoots $x_{L_{1}}$, but as the estimation of $k$ becomes closer to the actual value the sail approaches the equilibrium's $x$-position while the orbiting radius is reduced as the sail propulsion decays. Figure 11 shows a simulation of the sail trajectory for this case.

\section{Control Implementation}

So far in our discussion, we have neglected the actual implementation of control laws in the sail environment. We now show, through explicit discussion, that our methodology can be implemented into a standard sail design.

There are two ways in which the controller can be implemented. The sail can be made to spin with an average angular velocity and use the attitude control system to modulate the angular velocity needed to follow the nominal orbit. Thus, one of the sail body fixed axis is always pointing outward from the equilibrium 

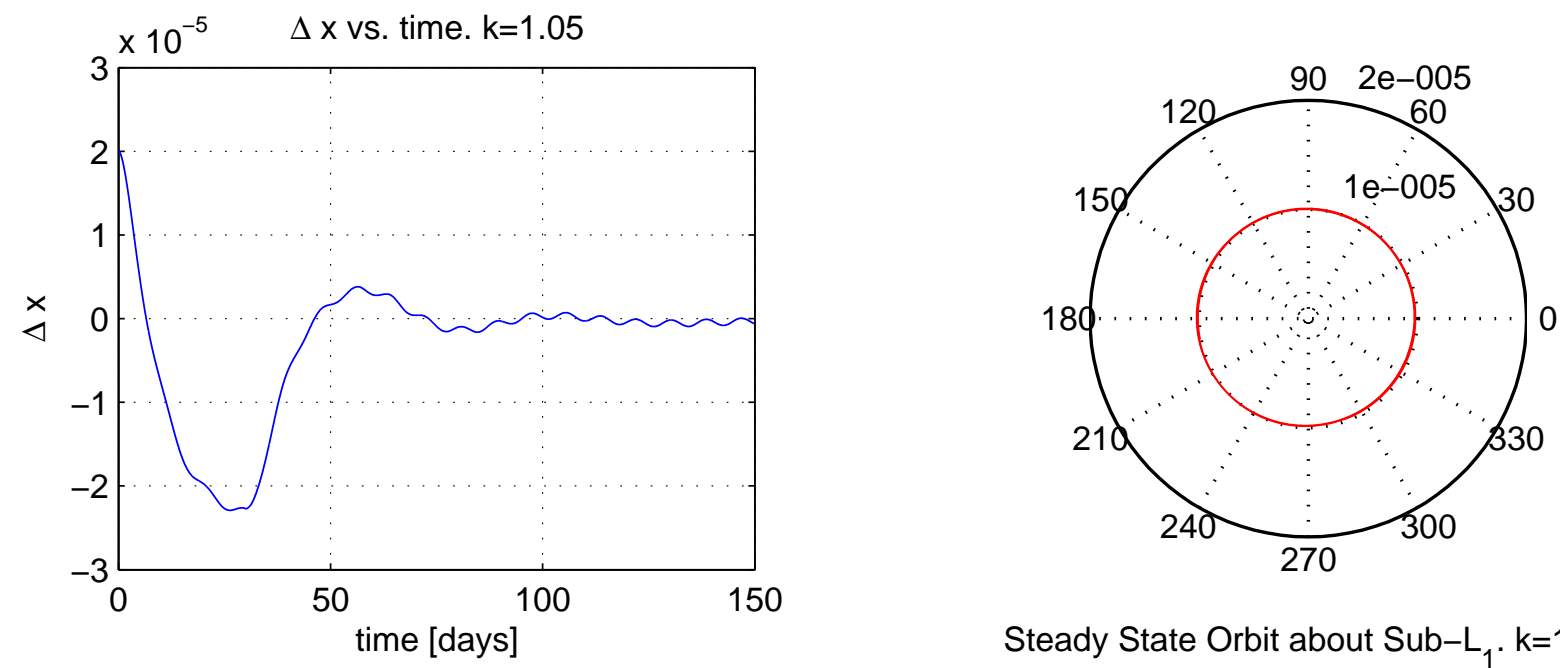

Steady State Orbit about Sub- $\mathrm{L}_{1} \cdot \mathrm{k}=1.05$
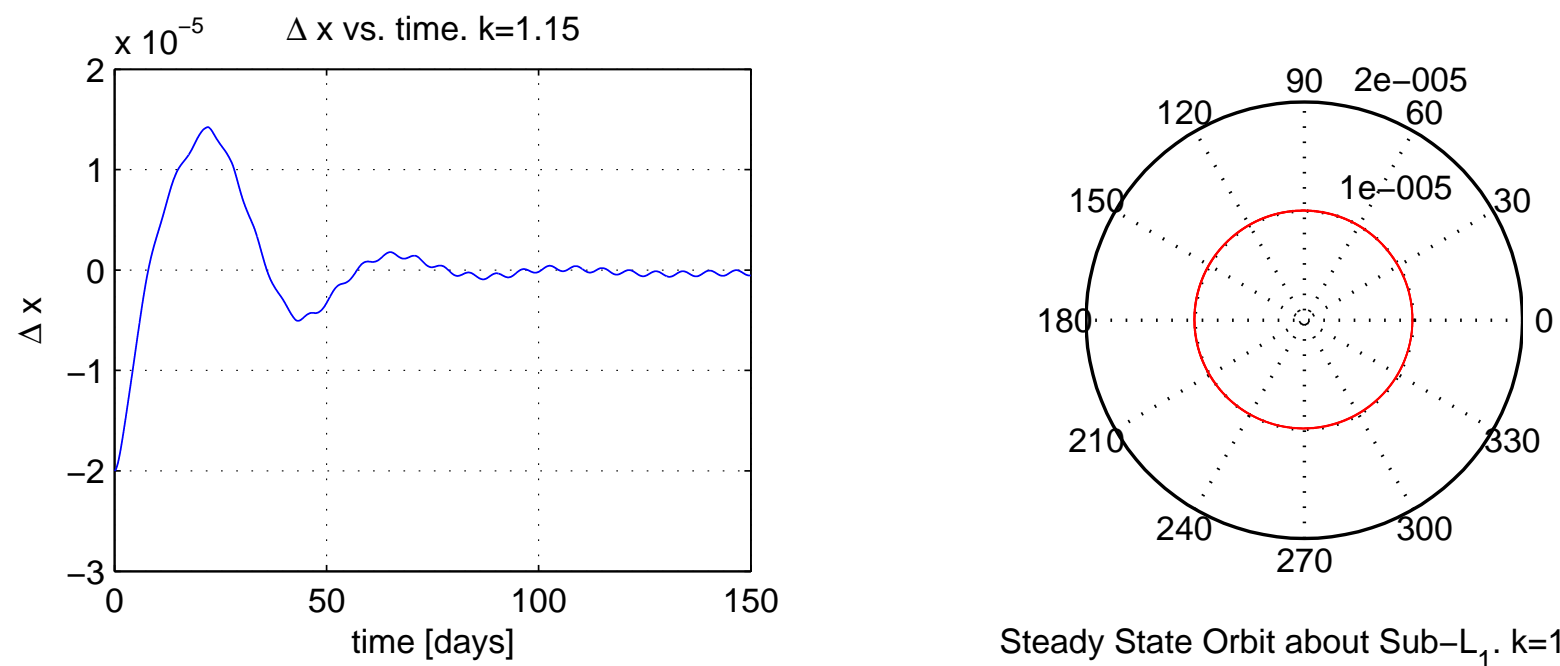

Steady State Orbit about Sub- $\mathrm{L}_{1} \cdot \mathrm{k}=1.15$

Figure 9. Adaptive Controller for the Simple PD-Controller.

point. In this case we choose the sail $y$ body-fixed axis to point away from the center of the sub- $L_{1}$ relative orbit. The angle $\alpha$ is controlled by exerting moments about the sail $x$-axis while $\delta$ is controlled by rotating the about sun-sail line. Another option is to change the sail attitude at every point in the orbit without exerting any moments about the sail normal axis. For this approach, $\alpha$ and $\delta$ are effected by a combination of moments about the sail $x$ and $y$ body-fixed axes. Both of these approaches control the angle $\alpha$ and $\delta$ to keep the sail in a quasi-circular orbit. In this paper we only consider the spinning case and we assumed that the sail behaves as a rigid body with moments of inertia axes coincident with the sail body-fixed axes.

The dynamics for a spinning rigid body are governed by Euler equations. Given a set of required angular velocities and their rates, the torques needed to achieved them are given by:

$$
\begin{aligned}
& M_{x}=I_{x} \dot{\omega}_{x}+\left(I_{z}-I_{y}\right) \omega_{y} \omega_{z} \\
& M_{y}=I_{y} \dot{\omega}_{y}+\left(I_{x}-I_{z}\right) \omega_{x} \omega_{z} \\
& M_{z}=I_{z} \dot{\omega}_{z}+\left(I_{y}-I_{x}\right) \omega_{x} \omega_{y}
\end{aligned}
$$

Where $M_{i}, I_{i}$, and $\omega_{i}$ are a moment, a moment of inertia, and an angular velocity about the sail bodyfixed $i$-axis, respectively. The angular velocities, in the sail body-fixed frame and with the sail $y$ body-fixed axis pointing outward from the sub- $L_{1}$ equilibrium point, can be obtained from the sail angle rates: 


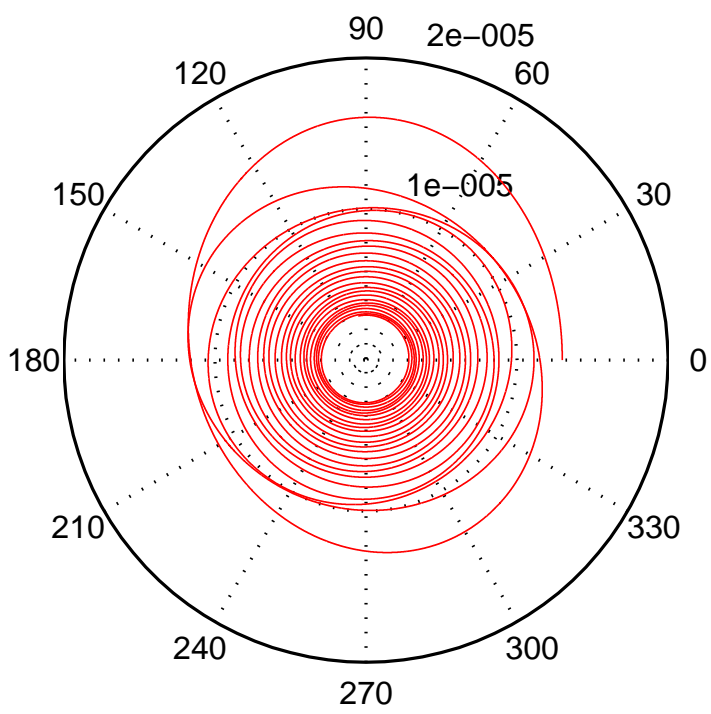

Sail Radius

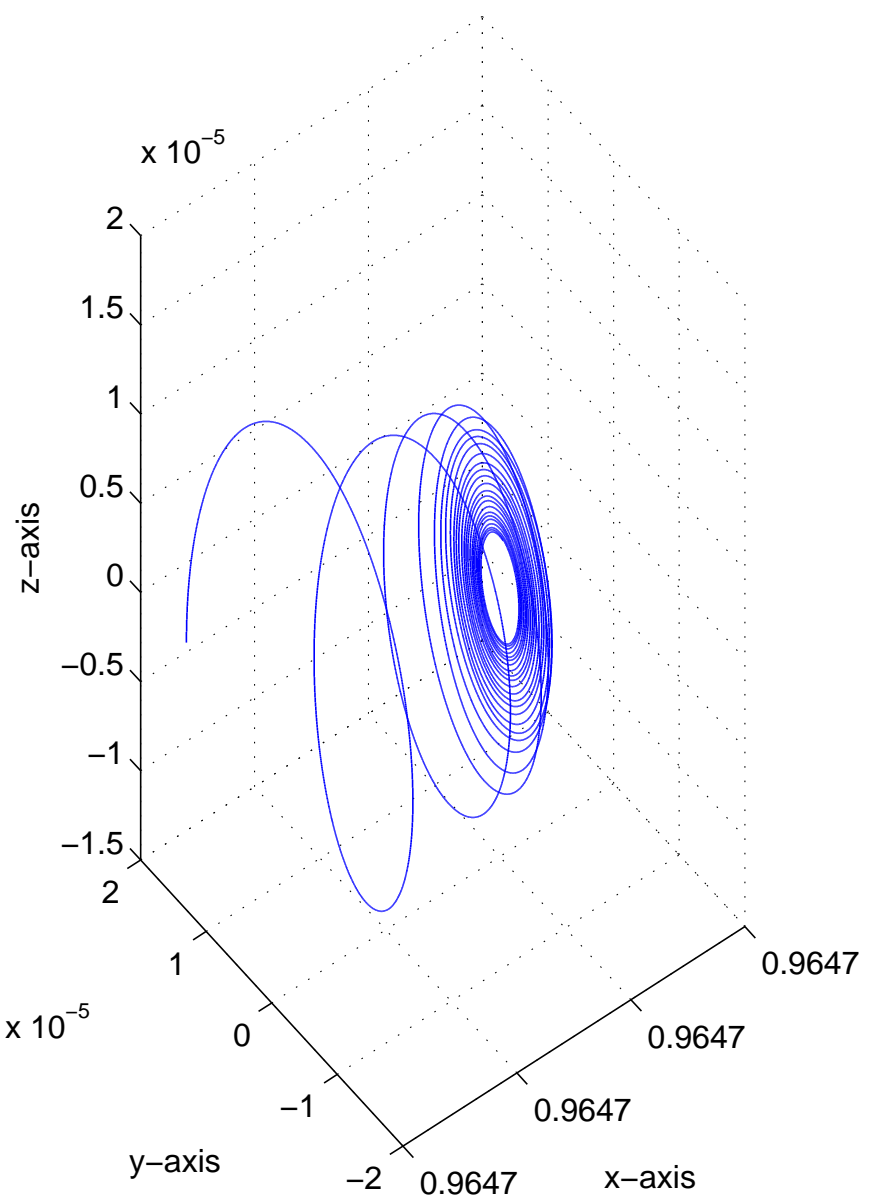

Figure 10. Solar Sail Under Degradation.

$$
\begin{aligned}
& \omega_{x}=\dot{\delta} \sin \alpha \\
& \omega_{y}=\dot{\alpha} \\
& \omega_{z}=-\dot{\delta} \cos \alpha
\end{aligned}
$$

Recall that $\delta=\theta+\bar{\delta}$. The angular rates are obtained from the controller commands and must be in radians per second. Taking a time derivative yields

$$
\begin{aligned}
& \dot{\omega}_{x}=\ddot{\delta} \sin \alpha+\dot{\delta} \dot{\alpha} \cos \alpha \\
& \dot{\omega}_{y}=\ddot{\alpha} \\
& \dot{\omega}_{z}=-\ddot{\delta} \cos \alpha-\dot{\delta} \dot{\alpha} \sin \alpha
\end{aligned}
$$

For a general sail, the moments needed are:

$$
\begin{aligned}
& M_{x}=I_{x}(\ddot{\delta} \sin \alpha+\dot{\delta} \dot{\alpha} \cos \alpha)-\left(I_{z}-I_{y}\right) \dot{\alpha} \dot{\delta} \cos \alpha \\
& M_{y}=I_{y} \ddot{\alpha}-\left(I_{x}-I_{z}\right) \dot{\delta}^{2} \cos \alpha \sin \alpha \\
& M_{z}=-I_{z}(\ddot{\delta} \cos \alpha-\dot{\delta} \dot{\alpha} \sin \alpha)+\left(I_{y}-I_{x}\right) \dot{\alpha} \dot{\delta} \sin \alpha
\end{aligned}
$$

These are the moments that need to be supplied to the sail by the attitude control system in order to follow the control commands. The moments can be generated by having control vanes, ${ }^{6}$ movable sail quadrants, or any other type of attitude control ${ }^{6} .{ }^{7}$ 

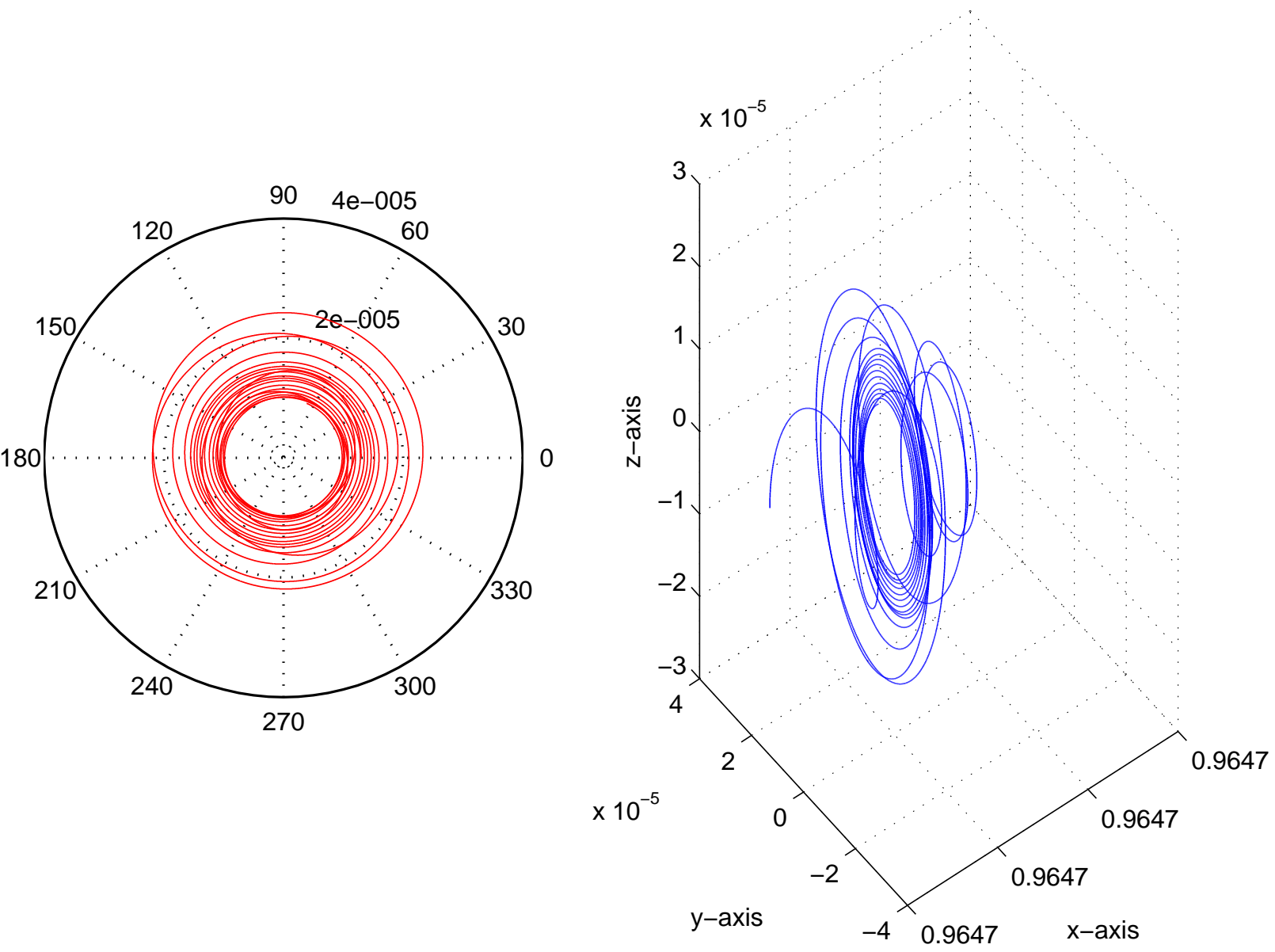

Figure 11. Solar Sail Under Degradation and Uncertainties.

For a sun-sail-line angle $\alpha$ that is almost constant, such as is the case of the last PD-controller developed, it can be assumed that $\ddot{\alpha}=0$ and $\dot{\alpha}=0$. Then, the control torques needed for the spinning the sail simplify to:

$$
\begin{aligned}
& M_{x}=I_{x} \ddot{\delta} \sin \alpha \\
& M_{y}=-\left(I_{x}-I_{z}\right) \dot{\delta}^{2} \cos \alpha \sin \alpha \\
& M_{z}=-I_{z} \ddot{\delta} \cos \alpha
\end{aligned}
$$

When the sail reaches steady-state $\ddot{\delta} \rightarrow 0$ and $\dot{\delta} \rightarrow$ constant. Thus the steady state moments are:

$$
\begin{array}{ll}
M_{x}=0 & \\
M_{y}= & \text { constant } \\
M_{z}=0 &
\end{array}
$$

\section{Conclusions}

In this paper a methodology for navigation of a solar sail with excess thrust was developed and applied to station-keeping a sail about a sub- $L_{1}$ point. Three controllers, one linear feedback and two proportionalderivative controllers, were developed to maintain the sail $x$-position at $x_{L_{1}}$ by enforcing a non-zero $\alpha$. One of the PD-controllers maintained the sail exactly at $x_{L_{1}}$ while the others made the sail oscillate about $x_{L_{1}}$. 
The excess thrust was used to orbit the sub- $L_{1}$ equilibrium. A control law based on the energy about the sub- $L_{1}$ point was developed to keep the sail at the desired orbit with $\delta$ as the controlling parameter.

A simple estimation methodology was developed to decrease errors in the initial estimate of the sail propulsion. The estimation procedure was based on the difference between $x_{L_{1}}$ and the sail steady state $x$-location when errors in the sail propulsion are present. This method was successful when applied to the case when the sail undergoes degradation.

Finally, a procedure for implementing these controllers was discussed. The implementation required a sail spinning about the sun-sail line. However, the steady state only required a single moment applied about the sail $y$ body-fixed axis.

\section{Acknowledgements}

The authors would like to acknowledge the support for this research from the Jet Propulsion Laboratory/California Institute of Technology, which is under contract with the National Aeronautics and Space Administration.

\section{References}

${ }^{1}$ Lura, F., and Hagelschuer, D., "Experiments in the Test Tacility Kobe for the Investigation of Degradation Effects of Thin Foil Samples for a Solar Sail Mission Concerning the Simultaneous Influence of Space Environment Properties," 22nd Space Simulation Conference, October 21-24, 2002.

${ }^{2}$ Lisano, M., Lawrence, D., Piggot, S. "Solar Sail Transfer Trajectory Design and Stationkeeping Control for Missions to the Sub-L1 Equilibrium Region," 15th AAS/AIAA Space Flight Mechanics Meeting, Copper Mountain, Colorado, January 23-27, 2005.

${ }^{3}$ Rios-Reyes, L. and Scheeres, D. J., "Generalized Model for Solar Sails," note in Journal of Spacecraft and Rockets, Vol 42 , No. 1, 2005, pp. 182-185.

${ }^{4}$ McInnes, C.R. [1999], Solar Sailing: Technology, Dynamics and Mission Applications, Springer-Praxis, Chichester, UK.

${ }^{5}$ Rios-Reyes, L. and Scheeres, D. J., "Applications of the Generalized Model for Solar Sails," AIAA Guidance, Navigation, and Control Conference and Exhibit, Providence, Rhode Island, August 2004. AIAA paper 2004-5434.

${ }^{6}$ Wie, B., "Dynamic Modeling and Attitude Control of Solar Sail Spacecraft: Part I," AIAA Guidance, Navigation, and Control Conference and Exhibit, 5-8 August 2002, Monterrey, California.

${ }^{7}$ Wie, B., "Dynamic Modeling and Attitude Control of Solar Sail Spacecraft: Part II," AIAA Guidance, Navigation, and Control Conference and Exhibit, 5-8 August 2002, Monterrey, California. 